\title{
ORIGINAL
}

\section{The effect of local anesthetic}

\section{continuous wound infusion for the prevention} of postoperative pneumonia after on-pump cardiac surgery with sternotomy: the
STERNOCAT randomized clinical trial

Julien Amour ${ }^{*} \mathbb{C}$, Bernard Cholley ${ }^{2}$, Alexandre Ouattara ${ }^{3}$, Dan Longrois ${ }^{4}$, Pascal Leprince ${ }^{5}$, Jean-Luc Fellahi ${ }^{6}$, Bruno Riou ${ }^{7}$, Sarah Hariri ${ }^{1}$, Christian Latrémouille ${ }^{8}$, Alain Rémy ${ }^{3}$, Sophie Provenchère ${ }^{4}$, Aude Carillion ${ }^{1}$, Paul Achouh ${ }^{8}$, Louis Labrousse ${ }^{9}$, Alexy Tran Dinh ${ }^{4}$, Nora Ait Hamou' ${ }^{1}$, Ahmed Charfeddine ${ }^{1}$, Alexandre Lafourcade ${ }^{10}$, David Hajage ${ }^{10}$, Adrien Bouglé ${ }^{1}$ and for the STERNOCAT investigators

\begin{abstract}
Purpose: Postoperative pain after cardiac surgery, exacerbated by cough and sternal mobilization, limits clearance of bronchopulmonary secretions and may predispose to postoperative pneumonia. In this study, we tested the ability of local anesthetic continuous wound infusion to prevent pneumonia after cardiac surgery with sternotomy and cardiopulmonary bypass (CPB) owing to better analgesia and bronchopulmonary drainage.

Methods: In this randomized, double-blind, placebo-controlled trial conducted in five academic centers, patients undergoing cardiac surgery with sternotomy and CPB were enrolled from February 2012 until November 2014, and were followed over 30 days. Patients were assigned to a 48 -h infusion $\left(10 \mathrm{ml} \mathrm{h}^{-1}\right)$ of L-bupivacaine $\left(12.5 \mathrm{mg} \mathrm{h}^{-1}\right)$ or placebo (saline) via a pre-sternal multiperforated catheter. Anesthesia and analgesia protocols were standardized. The primary end point was the incidence of pneumonia during the study period, i.e., until hospital discharge or 30 days. We hypothesized a 30\% reduction in the incidence of pneumonia.

Results: Among 1493 randomized patients, 1439 completed the trial. Pneumonia occurred in 36/746 patients (4.9\%) in the L-bupivacaine group and in 42/739 patients (5.7\%) in the placebo group (absolute risk difference taking into account center and baseline risk of postoperative pneumonia, $-1.3 \%[95 \% \mathrm{Cl}-3.4 ; 0.8] P=0.22)$. In the predefined subgroup of patients at high risk, L-bupivacaine decreased the incidence of pneumonia (absolute risk difference, $-5.6 \%[95 \% \mathrm{Cl}-10.0 ;-1.1], P=0.01)$.
\end{abstract}

\footnotetext{
*Correspondence: profulien.amour@gmail.com

1 Department of Anesthesiology and Critical Care Medicine, Pitié-

Salpêtrière Hospital, Institut de Cardiologie, Réanimation de Chirurgie

Cardiaque, Sorbonne Université, UMR INSERM 1166, IHU ICAN, Assistance

Publique-Hôpitaux de Paris (AP-HP), 47-83 Boulevard de I'Hôpital,

75013 Paris, France

Full author information is available at the end of the article
}

STERNOCAT group members are listed in the "Acknowledgements" section.

\section{鱼


Conclusions: After cardiac surgery with sternotomy, continuous wound infusion of L-bupivacaine failed to decrease the incidence of pneumonia. These findings do not support the use of local anesthetic continuous wound infusion in this indication. Further study should investigate its effect in high-risk patients.

Trial registration: EudraCT Number: 2011-003292-10; Clinicaltrials.gov Identifier: NCT01648777.

Keywords: Pneumonia, Cardiac surgery, Critical care medicine, Postoperative pain, Local anesthetics

\section{Introduction}

Pneumonia is a frequent complication in patients undergoing cardiac surgery with sternotomy and cardiopulmonary bypass $(\mathrm{CPB})[1-5]$. Although medical and surgical progress has consistently improved perioperative care, respiratory complications occur in 3-30\% [1-4] and represent one of the main causes of morbidity and mortality after cardiac surgery [6]. Postoperative pneumonia increases the risk of prolonged mechanical ventilation, renal replacement therapy, intensive care unit (ICU) and total hospital lengths of stay, as well as mortality $[2-4,6]$.

After cardiac surgery with sternotomy, postoperative pain plays an important role in impaired pulmonary function [5, 7-10]. Usually moderate to severe at rest, pain intensity increases strongly with iterative chest mobilization due to cough, deep breathing, and during active chest physiotherapy [9]. Because of changes in pulmonary mechanical properties, the colonization of the tracheobronchial tree by bacteria can evolve towards pneumonia $[2,5,11]$. Uncontrolled postoperative pain leads to increased pain distress [10], opioid consumption and its side effects, and pulmonary complications $[9,11-13]$. In this context, the use of local anesthetic continuous wound infusion $[14,15]$ through a multiperforated catheter within a multimodal analgesia strategy has been shown to improve postoperative analgesia after cardiac surgery with sternotomy [1, 14-17]. This therapeutic strategy may help to reduce the incidence of atelectasis and pulmonary disorders, offering the opportunity for better chest drainage and contributing to a decrease in the incidence of postoperative pneumonia.

The aim of this study was to test the ability of continuous wound infusion during the first $48 \mathrm{~h}$ of L-bupivacaine with multiperforated catheter to prevent postoperative pneumonia after cardiac surgery with sternotomy and CPB.

\section{Methods}

The study was approved by an independent National Research Ethics Committee (Comité de Protection des Personnes Ile de France VI; no. 83-11) and registered in EudraCT (2011-003292-10) and Clinicaltrials.gov (NCT01648777). The study protocol is available in the Online Supplement. The reporting of the study followed the Consolidated Standards of Reporting Trials (CONSORT) statement [18].

\section{Take-home message}

After cardiac surgery with sternotomy, continuous wound-infusion of L-bupivacaine slightly improves postoperative analgesia but does not reduce the incidence of pneumonia. These findings does not support the use of local anesthetic infusion in this indication.

\section{Participants}

Five academic cardiac surgical centers participated. Patients undergoing planned cardiac surgery with sternotomy and $\mathrm{CPB}$ were included after providing written informed consent.

Exclusion criteria were refusal to participate, age $<18$ years, pregnancy, emergency surgery, patient in whom fast-track cardiac anesthesia was not expected, moribund state, palliative care, decision of limitation of care, preoperative pneumonia, participation in another randomized trial, lack of national health care insurance, and allergy.

\section{Trial design and randomization}

This trial was a randomized, double-blind, two-arm, parallel-group, multicenter, placebo-controlled study (Fig. 1). The day before surgery, eligible patients were included and randomly assigned in a 1:1 ratio to receive a continuous infusion of L-bupivacaine (Chirocaïne ${ }^{\circledR}$, Abbott, Rungis, France) or matching placebo (saline) via a multiperforated catheter. The randomization list was computer-generated, balanced by blocks of variable and undisclosed size, and stratified by the center and by the baseline high or low risk of postoperative pneumonia. A patient was considered at high risk of pneumonia if he had two or more of the following factors: $\geq 70$ years old, previous cardiac surgery, chronic obstructive pulmonary disease with forced expiratory volume over $1 \mathrm{~s}$ (FEV1) $\leq 50 \%$ and/or a ratio of FEV-1/forced vital capacity $($ FVC $) \leq 70 \%$, cumulative smoking $>20$ cigarettes day $^{-1}$ for at least 20 years, renal dysfunction defined by an estimated preoperative creatinine clearance $<30 \mathrm{~mL} \mathrm{~min}{ }^{-1}$ (Cockcroft-Gault equation), diabetes mellitus requiring insulin therapy, and obesity (body mass index $\geq 30 \mathrm{~kg} \mathrm{~m}^{-2}$ ) or cachexia (body mass index $<15 \mathrm{~kg} \mathrm{~m}^{-2}$ ) [19-21]. 
Allocation concealment was achieved using a centralized, secure, interactive, web-response system accessible from each study center $\left(\right.$ Cleanweb $^{\circledR}$, Telemedecine Technologies S.A.S., Boulogne-Billancourt, France).

\section{Intervention}

L-Bupivacaine and placebo were prepared by an independent pharmacist. Both patient and medical team involved in the study were blinded to the allocated treatment.

A standardized protocol of antibiotic prophylaxis was performed, as recommended by national guidelines. Anesthesia induction and maintenance were performed with propofol and sufentanil in a target controlled infusion adapted to a bispectral index monitoring system $\left(\mathrm{BIS}^{\circledR}\right.$, Medtronic, MI, USA); the index was maintained at 40-60.

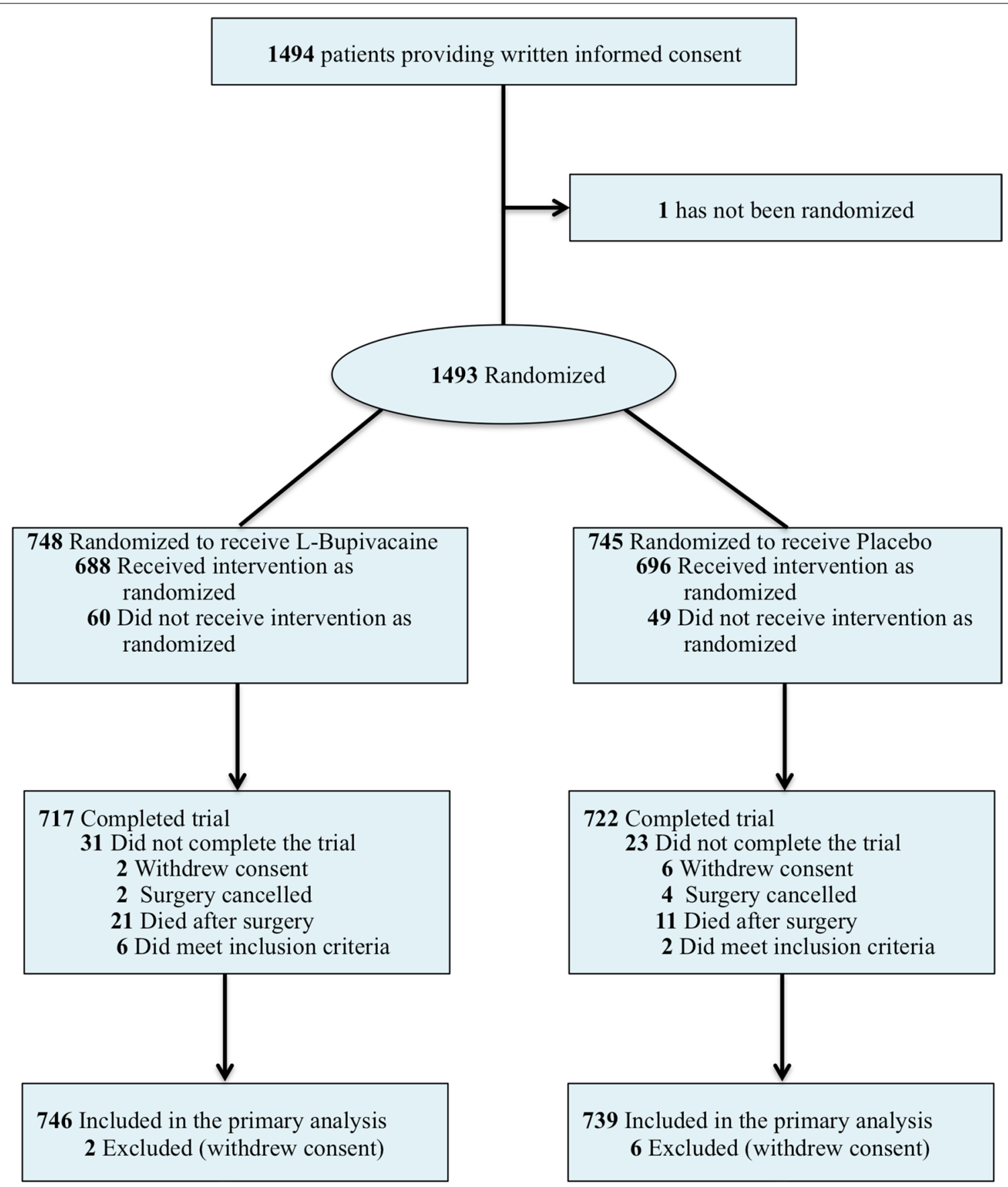

Fig. 1 Flow of participants through the study 
After sternal repair and before skin closure, a multiperforated wound catheter (Painfusor ${ }^{\circledR}$, Baxter, Maurepas, France) was positioned by the surgeon just above the sternal bone and below the adjacent sternal soft tissues in each patient. Depending on the study group, a $10-\mathrm{ml}$ bolus of L-bupivacaine $(50 \mathrm{mg}$ ) or saline was administered through the catheter (day 0 ). Then, a continuous infusion $\left(10 \mathrm{ml} \mathrm{h}^{-1}\right)$ of L-bupivacaine $\left(12.5 \mathrm{mg} \mathrm{h}^{-1}\right)$ or saline was started for $48 \mathrm{~h}$ using a specific elastomeric pump (Varicon ${ }^{\circledR}$, Wym France, Paris, France). As recommended in the fast-track cardiac anesthesia strategy, the tracheal extubation was ideally performed within $6 \mathrm{~h}$ after the end of skin closure. Initial intravenous morphine sulfate titration was started until the numerical rating scale (NRS) was $\leq 3$. Then, morphine was administrated with a patient-controlled analgesia device (PCA, Injectomat Master PCA ${ }^{\circledR}$, Fresenius Kabi, Sèvres, France). Total cumulative consumption of morphine, including initial intravenous titration, was recorded for each patient during the first $48 \mathrm{~h}$. Both FEV-1 and NRS were assessed at rest and after mobilization during standardized daily physiotherapy sessions during the first $48 \mathrm{~h}$. Between physiotherapy sessions, an incentive spirometer (Respiflo $^{\circledR}$, Tyco Kendall Healthcare, Elancourt, France) was made available to the patient. A systematic non-invasive ventilation session was performed for at least $1 \mathrm{~h}$ per day in ICU. None of our patients was treated with thoracic epidural analgesia. The ICU discharge decision was left to the discretion of the physicians in charge.

\section{Outcomes}

The primary end point was the incidence of pneumonia during the study period, i.e., until hospital discharge or 30 days. If a patient had multiple episodes of pneumonia, only the first one was taken into account. The International Sepsis Forum Definition of Infection in the ICU Consensus Conference was used for the diagnosis of infection [22-24]. The retrospective diagnosis of infection was determined independently by three experts, following analysis of the complete medical file [25]. Experts were blinded with respect to patient allocation. The agreement between the three experts was calculated using Fleiss' kappa score.

The secondary end points, assessed during the study period, were ICU-free days defined as the number of days alive until ICU discharge (quoted as 0 if death occurred), hospital length of stay, renal replacement rate, renal replacement therapy-free days, mechanical ventilationfree days, tracheal reintubation, total morphine sulfate consumption, NRS at rest and after mobilization, FEV-1 at rest and after mobilization, septic shock, antibioticfree days, stroke, and major adverse cardiovascular events (MACCE, defined as the occurrence of stroke and cardiac damage). Myocardial damage was reflected by the highest troponin value measured until the first $24 \mathrm{~h}$. Laboratory explorations were not centralized. All-cause mortality was assessed until 30 days.

The drug safety was assessed during the study period by recording the incidence of seizures, high-degree atrioventricular blocks, ventricular tachycardia or ventricular fibrillation requiring cardioversion, and cardiac arrest. In the specific case of mediastinitis, because it may occur beyond day 30, we performed an additional post hoc analysis until 3 months after surgery by the three experts involved in the retrospective diagnosis of infectious events [24].

\section{Sample size}

The sample size estimation was based on an assumed event rate of $18 \%$ in the placebo group (median value of the incidence of pneumonia estimated at the different cardiac surgery centers involved, prior to the study). To demonstrate a decrease in the incidence of pneumonia by $30 \%$ in the intervention group, with an alpha risk of 0.05 and a beta risk of 0.20 , we calculated that 734 patients per group had to be included [26]. To account for potential patient loss on follow-up, we chose to include 750 patients per group, i.e., a total of 1500 patients.

\section{Statistical analysis}

An intention-to-treat analysis was performed on all randomized patients, except those who withdrew their consent. In addition, a per-protocol post hoc analysis was performed excluding patients in each group who did not receive the intervention planned by the randomization.

For primary outcome analysis, a generalized linear model (binomial family and identity link function) with adjusted stratification factors (center and baseline risk of pneumonia) was performed to estimate the risk difference of pneumonia between the two groups and its $95 \%$ confidence interval $(95 \% \mathrm{CI})$. In addition, a predefined subgroup analysis was also performed according to the baseline risk of pneumonia, using the same model but including an interaction term between this characteristic and the treatment group.

To handle missing outcome values, three analytical methods were used: a "complete-case" analysis [27], a "worst-best-case" analysis (for which it is assumed that all participants with missing outcome in the L-bupivacaine group have had postoperative pneumonia; and all participants with missing outcome in the placebo group have had no postoperative pneumonia) [27], and a "multiple imputation" analysis. Briefly, multiple imputations 
were performed using ten imputed data sets, and results from each imputed data set were pooled using Rubin's rules [28]. The multiple imputation procedure was fully described in supplementary materials. Only the worstbest-case analysis and the multiple imputation analysis include all randomized patients without consent withdrawn, and could be considered as intention-to-treat analyses.

Incidence of pneumonia over time was depicted as a cumulative incidence curve for each group. Death without pneumonia was taken into account as a competing risk, and a Gray's test was used for group comparison.

For secondary end point analysis, quantitative variables were expressed as mean (SD) or median (IQR) in nonnormally distributed variables. Comparisons between the two groups were performed using the Student's $t$ test or Wilcoxon rank sum test as appropriate. Mean difference between the two groups with 95\% CI was reported for normally distributed variables. Qualitative variables were expressed as number (percentage). Comparisons between two groups were performed using the $\chi^{2}$ Pearson test or the Fisher test when appropriate. Absolute risk difference (with $95 \% \mathrm{CI}$ ) was also reported.

All $P$ values were two-tailed, and a $P<0.05$ was considered significant. Sample size calculation and statistical analyses were performed by independent statisticians (AL, DH) using the SAS version 9.4 (SAS Institute, Cary, N.C.).

\section{Results}

The randomization and the follow-up of the patients are shown in Fig. 1. A total of 1494 were eligible and 1493 were randomized from March 2012 until November 2014 and followed during 30 days (last follow-up December 26, 2014). The last seven patients were not included for funding reasons.

A total of 1439 patients completed the trial (Fig. 1) and 1384 received interventions as randomized. Overall, $60(8.0 \%)$ patients in the L-bupivacaine group and $49(6.6 \%)$ in the placebo group did not receive the treatment. In addition, $2(0.3 \%)$ patients in the L-bupivacaine group and $6(0.8 \%)$ in the placebo group withdrew consent. Therefore, 746 (99.7\%) patients in the L-bupivacaine group and $739(99.2 \%)$ in the placebo group were analyzed on an intention-to-treat 49 (6.6). The L-bupivacaine and the placebo groups were well balanced as shown in the baseline characteristics (Table 1). The surgical procedures are described in Table 2.

Overall, the number of missing values for the primary outcome was $10(1.3 \%)$ in the L-bupivacaine group and $6(0.8 \%)$ in placebo group. In the complete-case analysis, postoperative pneumonia occurred in $36 / 736$ (4.9\%) patients in the L-bupivacaine group and in $42 / 733$ (5.7\%) patients in the placebo group during the study period (absolute risk difference taking into account a center effect and the baseline risk of pneumonia effect, $-1.3 \%$ [95\% CI -3.4 to 0.8 ], $P=0.22$ ). The worst-best-case analysis and the multiple imputation analysis provided similar conclusions. Most cases of pneumonia occurred during the first 10 days (Fig. 2). Agreement between experts was good as shown by Fleiss' kappa value measured at 0.80 [95\% CI 0.77-0.82]. In per-protocol-analysis, 688 (95.9\%) patients in the L-bupivacaine group and 695 $(96.3 \%)$ in the placebo group were analyzed. The primary end point was not significantly different $(P=0.84)$.

In the secondary end point analyses, total consumption of morphine during the first $48 \mathrm{~h}$ was slightly reduced in the L-bupivacaine group in comparison with the placebo group, respectively 24 (11-43) and 27 (13-48) mg $(P=0.01)$ (Table 3$)$. At rest as well as after mobilization, the quality of analgesia was not significantly different between groups as shown by NRS (Table 3). At rest as well as after mobilization, pulmonary capacity was not significantly different between groups as shown by FEV-1 (Table 3). In addition, the rate of additional surgical incision associated with the sternotomy was not different between groups $(P=0.76)$ (Table 2$)$.

ICU-free days, hospital length of stay, renal replacement rate, renal replacement therapy-free days, mechanical ventilation-free days, tracheal reintubation, antibiotic-free days, septic shock, stroke, MACCE, and all-cause mortality until 30 days were not significantly different between groups in the intention-to-treat analysis (Table 3) or per-protocol analysis (eTable 1 ).

The incidence of serious adverse events was not different between groups in the intention-to-treat analysis (Table 3) or per-protocol analysis (eTable 1).

In the subgroup of patients at high risk of pneumonia, L-bupivacaine significantly decreased the incidence of pneumonia in comparison with the placebo group in the complete-case analysis: 9/233 (3.9\%) versus 20/230 (8.7\%) (absolute risk difference, $-5.6 \%$ [95\% CI $-10.0 ;-1.2] ; P=0.01$ ) (Fig. 3a). The multiple imputation analysis (eFig. 1 ) and the worst-bestcase analysis (eFig. 2) showed a similar pattern, but the difference did not reach significance among high-risk patients in the worst-best-case analysis. There was no difference in terms of total morphine consumption and NRS during the first $48 \mathrm{~h}$. Nevertheless, FEV-1 variations after mobilization were significantly improved by L-bupivacaine (eTable 2). Lengths of stay in ICU and in hospital, ventilator-free days, tracheal reintubation, renal replacement-free days, antibiotic-free days (eTable 2), and all-cause mortality until 30 days (Fig. 3b) were not different between groups. 
Table 1 Baseline patient characteristics

\begin{tabular}{|c|c|c|}
\hline & L-Bupivacaine $(n=746)$ & Placebo $(n=739)$ \\
\hline Age, mean (SD), year & $67(13)[0]$ & $66(13)[0]$ \\
\hline Male sex & $526(71)[0]$ & $542(73)[0]$ \\
\hline Body mass index, mean (SD), $\mathrm{kg} \mathrm{m}^{-2}$ & $27(4)[0]$ & $27(4)[0]$ \\
\hline ASA class ${ }^{a}$ & {$[0]$} & {$[0]$} \\
\hline $1-2$ & $175(23)$ & $188(25)$ \\
\hline 3 & $554(74)$ & $540(73)$ \\
\hline 4 & $17(2)$ & $11(1)$ \\
\hline Logistic EuroSCORE, ${ }^{b}$ median (IQR), \% & $3.3(1.8-6.1)[209]$ & $4.00(2.0-6.0)[201]$ \\
\hline LVEF, median (IQR), \% & $60(55-67)[51]$ & $60(55-67)[53]$ \\
\hline Diabetes mellitus & $145(19)[0]$ & $161(22)[0]$ \\
\hline Type I & $10(7)$ & $9(6)$ \\
\hline Type ॥ & $135(93)$ & $152(95)$ \\
\hline Hypertension & $495(66)[0]$ & $473(64)[0]$ \\
\hline Ischemic cardiomyopathy & $322(43)[0]$ & $351(48)[0]$ \\
\hline Creatinine clearance, median (IQR), $\mathrm{mL} \mathrm{min}^{-1}$ & $80(62-104)[0]$ & $83(62-105)[0]$ \\
\hline Patients on long-term dialysis & $8(1)[0]$ & $7(1)[0]$ \\
\hline Dyslipidemia & $460(62)[0]$ & $477(65)[0]$ \\
\hline High risk status of postoperative pneumonia & $237(32)[0]$ & $232(32)[0]$ \\
\hline Age $>70$ years old & $324(43)$ & $298(40)$ \\
\hline Previous cardiac surgery & $48(6)$ & $50(7)$ \\
\hline Severe chronic obstructive pulmonary disease & $128(17)$ & $129(17)$ \\
\hline Smoker $\geq 20$ pack-years & $144(19)$ & $153(21)$ \\
\hline Body mass index $<15$ or $>30 \mathrm{~kg} \mathrm{~m}^{-2}$ & $150(20)$ & $153(20)$ \\
\hline Creatinine clearance $<30 \mathrm{~mL} \mathrm{\textrm {min } ^ { - 1 }}$ & $24(3)$ & $15(2)$ \\
\hline Type II diabetes requiring insulin & $32(4)$ & $40(5)$ \\
\hline Preoperative medication & {$[0]$} & {$[0]$} \\
\hline Beta-blockers & $405(54)$ & $375(51)$ \\
\hline Statins & $437(59)$ & $442(60)$ \\
\hline Anticoagulant therapy (INR > 1.5) & $5(1)$ & $8(1)$ \\
\hline \multicolumn{3}{|l|}{ Antiplatelet therapy } \\
\hline Aspirin & $396(53)$ & $416(56)$ \\
\hline Clopidogrel & $92(12)$ & $88(12)$ \\
\hline Prasugrel & $3(0.4)$ & $5(0.7)$ \\
\hline Corticosteroids & $32(4)$ & $18(2)$ \\
\hline
\end{tabular}

Values are expressed as number (percentage), unless otherwise indicated, and followed by [missing data]

$S D$ standard deviation, ASA American Society of Anesthesiology, IQR interquartile range, INR international normalized ratio, LVEF left ventricular ejection fraction

a ASA class 1, normal healthy patient; class 2, patient with mild systemic disease; class 3, patient with severe systemic disease; class 4 , patient with severe systemic disease that is a constant threat to life; and class 5, moribund patient who is not expected to survive without the operation

b Logistic Euroscore is a risk model that provides the risk of death after cardiac surgery

\section{Discussion}

In this randomized placebo-controlled trial, L-bupivacaine continuous wound infusion for the first $48 \mathrm{~h}$ after planned cardiac surgery with sternotomy and CPB did not significantly decrease the incidence of pneumonia during the study period in comparison with placebo and contributes only very slightly to better analgesia. In a subgroup of high-risk patients, L-bupivacaine may decrease the incidence of pneumonia.
After cardiac surgery, pulmonary dysfunction frequently leads to a decrease in pulmonary capacity associated with atelectasis and ventilatory disorders [1-3] in which postoperative pain plays an important role [5, 7-9]. The objective of a multimodal approach including the local anesthetic wound infusion is to improve analgesia [10] and then to improve chest movement, coughing, deep breathing, mobilization, and active chest physiotherapy [9], to decrease opioid consumption 
Table 2 Surgical procedures

\begin{tabular}{|c|c|c|}
\hline & L-Bupivacaine $(n=746)$ [missing data $=11]$ & $\begin{array}{l}\text { Placebo }(n=739) \\
\text { [missing data }=8 \text { ] }\end{array}$ \\
\hline CABG & $334(45)$ & $342(47)$ \\
\hline Bilateral internal thoracic artery harvesting & $208(60)$ & $218(64)$ \\
\hline Left internal thoracic artery harvesting only & $120(36)$ & $112(33)$ \\
\hline \multicolumn{3}{|c|}{ Other surgical incision associated with the sternotomy } \\
\hline Saphenous graft & $99(13)$ & $102(14)$ \\
\hline Radial artery graft & $1(0)$ & 0 \\
\hline Carotid endarterectomy & $7(1)$ & $8(1)$ \\
\hline Aortic valve replacement & $320(44)$ & $298(41)$ \\
\hline Mitral valve repair & $70(10)$ & $68(9)$ \\
\hline Mitral valve replacement & $48(7)$ & $49(7)$ \\
\hline Tricuspid valve repair & $31(4)$ & $31(4)$ \\
\hline Ascending aortic surgery & $77(10)$ & $78(11)$ \\
\hline Other procedures & $13(2)$ & $17(2)$ \\
\hline Complex cardiac surgery & $153(21)$ & $148(20)$ \\
\hline CPB time, median (IQR), min & $77(62-103)$ & $77(61-103)$ \\
\hline Aortic cross-clamp time, median (IQR), min & $58(45-78)$ & $59(45-77)$ \\
\hline Total dose of sufentanil, median (IQR), $\mu \mathrm{g}$ & $138(112-165)$ & $136(110-165)$ \\
\hline Total ventilation time, median (IQR), min & $600(480-780)$ & $600(480-816)$ \\
\hline
\end{tabular}

Values are expressed as number (percentage) unless otherwise indicated

$C A B G$ coronary artery bypass graft, $C P B$ cardiopulmonary bypass, IQR interquartile range

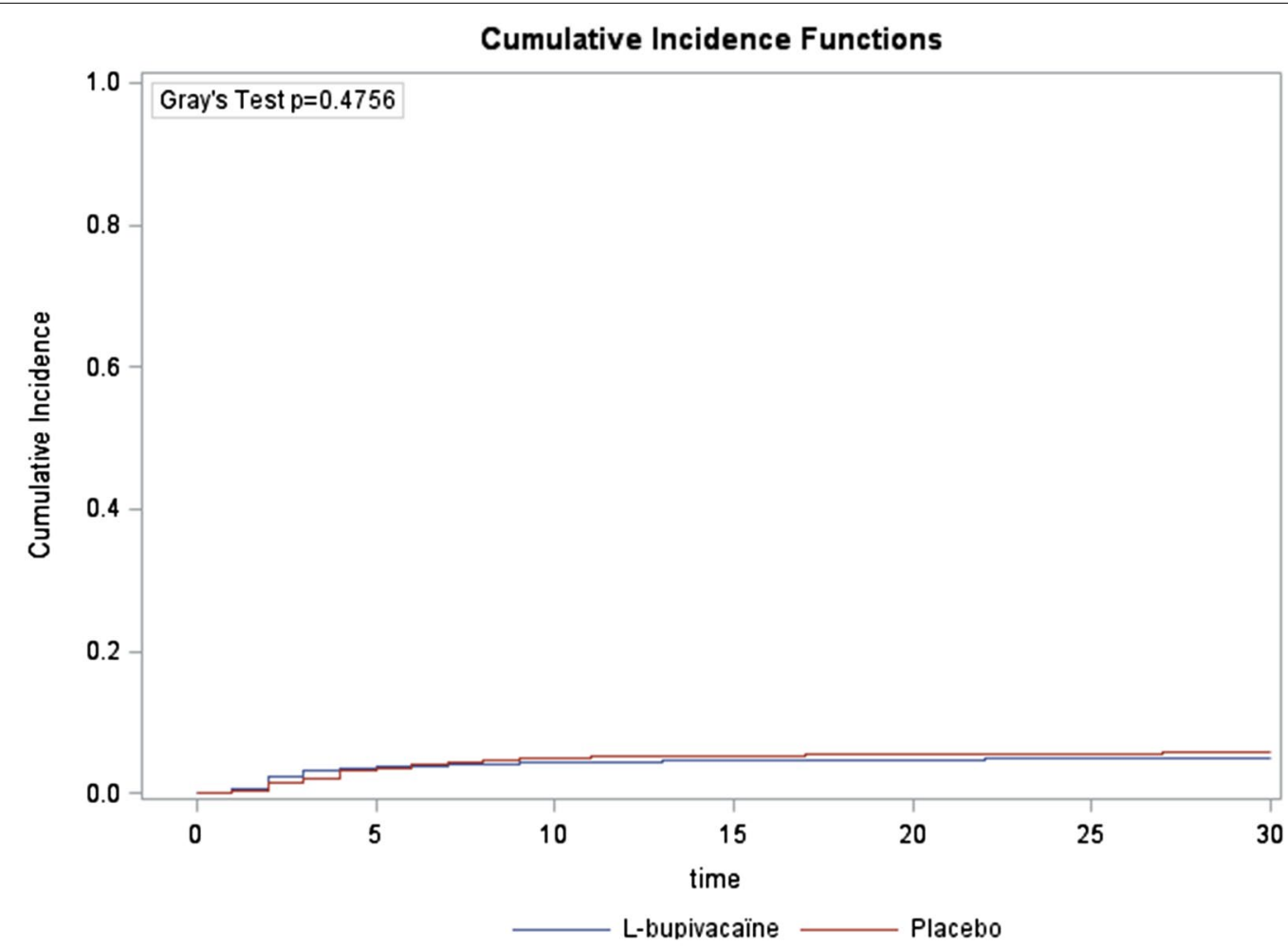

Fig. 2 Comparison of the cumulative incidence of postoperative pneumonia over time between the L-bupivacaine group and the placebo group during the first 30 postoperative days 
Table 3 Secondary end points during the study period in analysis population (until hospital discharge or 30 days) (complete-case analysis)

\begin{tabular}{|c|c|c|c|c|}
\hline & L-Bupivacaine $(n=746)$ & Placebo $(n=739)$ & Mean difference, $\%[95 \%$ ] & $P$ value \\
\hline \multicolumn{5}{|l|}{ Mortality } \\
\hline All-cause mortality in the first 30 days & $21(3)[7]$ & $11(2)[6]$ & $1.3[-0.2 ; 2.8]$ & 0.15 \\
\hline In-hospital death & $20(3)[7]$ & $11(2)[6]$ & $1.2[-0.3 ; 2.7]$ & 0.16 \\
\hline \multicolumn{5}{|l|}{ Length of stay, and treatment outcomes during study period } \\
\hline Length of hospital stay, median (IQR), day & $10(8-14)[10]$ & $10(8-14)[6]$ & & $0.08^{\$}$ \\
\hline Length of ICU stay, median (IQR), day & $2(1-4)[10]$ & $2(1-5)[6]$ & & $0.59^{\$}$ \\
\hline ICU-free days, median (IQR), day & $28(25-29)[7]$ & $28(25-29)[6]$ & & $0.88^{\$}$ \\
\hline Tracheal reintubation & $68(9)[10]$ & $54(7)[6]$ & $1.9[-1 ; 4.7]$ & 0.07 \\
\hline Septic shock & $2(0.3)[10]$ & $2(0.3)[6]$ & $-0.0[-0.5 ; 0.5]$ & $0.99^{\$}$ \\
\hline Ventilator-free days, median (IQR), day & $30(29-30)[7]$ & $30(29-30)[6]$ & & 0.21 \\
\hline De novo renal replacement therapy & $25(3)[10]$ & $27(4)[6]$ & $-0.3[-2.2 ; 1.6]$ & 0.85 \\
\hline Renal replacement therapy-free days, median (IQR), day & $30(30-30)[7]$ & $30(30-30)[6]$ & & $0.55^{5}$ \\
\hline Antibiotics $\geq 1$ day & $140(19)[10]$ & $160(21)[6]$ & $-2.8[-6.9 ; 1.3]$ & 0.24 \\
\hline Antibiotic-free days, median (IQR), day & $30(30-30)[10]$ & $30(30-30)[6]$ & & $0.44^{\lessgtr}$ \\
\hline MACCE & $29(4)[0]$ & $29(4)[0]$ & $-0.0[-2.0 ; 1.9]$ & 0.92 \\
\hline Stroke & $2(0.3)[10]$ & $5(0.7)[6]$ & $-0.4[-1.1 ; 0.3]$ & $0.28^{5}$ \\
\hline \multicolumn{5}{|l|}{ Postoperative pain outcomes } \\
\hline Total morphine dose during the first $48 \mathrm{~h}$, median (IQR), mg & $24(11-43)[27]$ & $27(13-48)[25]$ & & $0.01^{\$}$ \\
\hline NRS at day 1 , at rest, mean (SD), $\mathrm{cm}$ & $34(22)[152]$ & $35(23)[139]$ & $-1.4[-3.9 ; 1.6]$ & 0.31 \\
\hline NRS variations at day 1 after mobilization, mean (SD), cm & $4(15)[154]$ & $4(14)[147]$ & $-0.8[-2.4 ; 0.8]$ & 0.35 \\
\hline NRS at day 2 , at rest, mean (SD), $\mathrm{cm}$ & $29(23)[202]$ & $31(23)[180]$ & $-1.4[-4.1 ; 1.3]$ & 0.35 \\
\hline NRS variations at day 2 after mobilization, mean (SD), $\mathrm{cm}$ & $1(12)[211]$ & $1(13)[192]$ & $0.3[-1.2 ; 1.8]$ & 0.66 \\
\hline FEV-1 at day 1 , at rest, mean (SD), $\mathrm{L} \mathrm{min}^{-2}$ & $173(64)[154]$ & $167(67)[145]$ & $6.0[-1.5 ; 13.4]$ & 0.09 \\
\hline $\begin{array}{l}\text { FEV- } 1 \text { variations at day } 1 \text { after mobilization, mean (SD), L } \\
\text { min }^{-2}\end{array}$ & $23(40)[155]$ & $20(36)[154]$ & $3.5[-0.8 ; 7.8]$ & 0.07 \\
\hline FEV-1 at day 2 , at rest, mean (SD), $\mathrm{L} \mathrm{min}^{-2}$ & $173(66)[205]$ & $173(69)[187]$ & $0.3[-7.7 ; 8.3]$ & 0.89 \\
\hline $\begin{array}{l}\text { FEV-1 variations at day } 2 \text { after mobilization, mean (SD), L } \\
\text { min }^{-2}\end{array}$ & $16(31)[210]$ & $15(27)[196]$ & $1.5[-2.0 ; 5.0]$ & 0.37 \\
\hline \multicolumn{5}{|l|}{ Safety outcomes } \\
\hline Mediastinitis in the first 30 days & 27(4) [10] & $29(4)[10]$ & $-0.3[-2.2 ; 1.6]$ & 0.41 \\
\hline Mediastinitis in the first 3 months & $28(4)[10]$ & $31(4)[6]$ & $-0.4[-2.4 ; 1.6]$ & 0.48 \\
\hline Seizures during study period & $3(0.4)[0]$ & $4(0.1)[0]$ & $0.3[-0.2 ; 0.8]$ & $0.62^{\$}$ \\
\hline High-degree atrioventricular block during study period & $11(2)[0]$ & $9(1)[0]$ & $0.3[-0.9 ; 1.4]$ & 0.98 \\
\hline $\begin{array}{l}\text { Major ventricular arrhythmia treated by cardioversion during } \\
\text { study period }\end{array}$ & $12(2)[0]$ & $10(1)[0]$ & $0.3[-1.0 ; 1.5]$ & $0.68^{\$}$ \\
\hline Cardiac arrest during study period & $8(1)[0]$ & $11(1)[0]$ & $-0.4[-1.6 ; 0.7]$ & 0.53 \\
\hline
\end{tabular}

Values are expressed as number (percentage), unless otherwise indicated, and followed by [missing data]

$S D$ standard deviation, IQR interquartile range, ICU intensive care unit, MACCE major adverse cardiac and cardiovascular events, NRS numerical rating scale, FEV-1 forced expiratory volume in $1 \mathrm{~s}$

\$ All statistical tests were adjusted on stratification factors (center and baseline risk of pneumonia) except (indicated with ${ }^{\$}$ ) in case of deviation from the normality assumption or convergence issues with the multivariate model

and its related side effects, and then to improve patient outcomes [11]. The use of thoracic epidural analgesia, which has been shown to be an efficient analgesic therapy in thoracic surgery, is still a matter of debate in cardiac surgery because of the increased risk of perimedullary hematoma associated with the high concentration of anticoagulation therapy required for CPB [29, 30]. In parallel, small studies suggested the safety of local anesthetic continuous wound infusion in reducing postoperative pain [14, 15]. A meta-analysis of 42 heterogeneous studies involving 2141 patients undergoing various types of surgery revealed the superiority of analgesia combining morphine and local anesthetic wound infusion compared with morphine alone [31]. This results in a $40 \%$ decrease in overall morphine consumption. In cardiac surgery, despite the small size of 


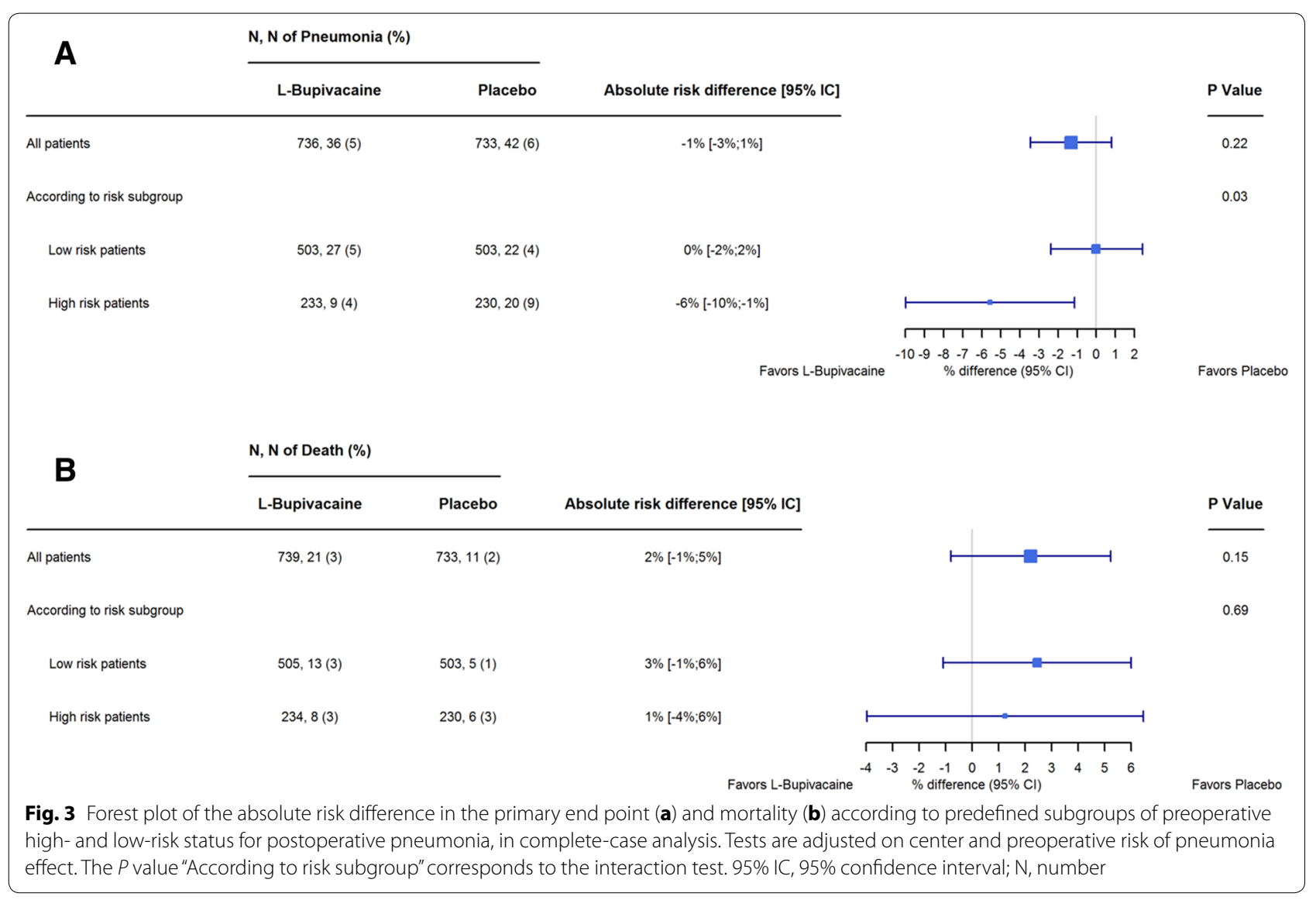

the few studies carried out, the results suggested a significant beneficial analgesic effect of local anesthetic wound infusion [1, 14-16]. In a recent small trial performed in a single center, Eljezi et al. observed a better effect of respiratory physiotherapy, despite the lack of enhancement of spirometric variables, in parallel with a significant decrease in pain, both at rest and during chest mobilization [1]. These results were associated with a significant decrease in the incidence of all-cause pulmonary complications, without being able to conclude on the incidence of pneumonia. In the current study, with a much larger sample size and a doubleblind intervention, the incidence of pneumonia during the study period was similar between groups. Despite a slight decrease in morphine consumption during the first $48 \mathrm{~h}$, pain score (NRS) and lung capacity (FEV-1) were not improved by L-bupivacaine. The secondary end points were not improved. Overall, when a large double-blind trial is provided, the slight beneficial analgesic effect of $\mathrm{L}$-bupivacaine is not enough to improve postoperative respiratory function and the incidence of pneumonia.

In high-risk patients, in complete-case analysis as well as in multiple imputation analysis, our results suggest that L-bupivacaine may decrease the incidence of pneumonia. The potential beneficial effect of L-bupivacaine may be explained by better chest mobilization and improved lung capacity as shown by the increase of FEV-1 up to $10 \%$ after mobilization in the L-bupivacaine group. The fact that NRS and total morphine consumption did not improve in this subgroup suggests the highly subjective and variable nature of these parameters from one individual to another. Regardless, improved chest mobilization indirectly reflects improved analgesia. Of course, further randomized study focused on high-risk patients should be performed to confirm this hypothesis because, on the one hand, this is a subgroup statistical analysis and, on the other hand, two of the statistical analyses performed, complete-case and multiple imputation, concluded a significant difference between L-bupivacaine and placebo groups but the worst-best-case analysis, the most unfavorable regarding the experimental group, did not.

Overall, despite the implantation of the catheter in the sternal wound, we did not observe any difference in mediastinitis rate between groups. The relatively high rate of mediastinitis in the current study (4\%) is consistent with the high proportion of CABG performed with 
bi-mammary artery grafts and the high proportion of diabetic and obese patients [32, 33].

This study has several limitations. The first, and obviously the most important, is the overestimation of the expected incidence of pneumonia. The observed incidence was very much lower (5.4\%) than hypothesized for the sample size calculation (18\%). Unfortunately, our hypothesis was based on the literature data at the time we wrote the study protocol in 2010, which was quite wide-ranging (3-30\%) [1-4]. The high heterogeneity in the diagnostic criteria of pneumonia used in these different studies may contribute to this large range. In addition, because pneumonia is a difficult diagnosis and overestimated in daily practice, its incidence measured for the sample size calculation in our five centers was also probably overestimated. To support this hypothesis, during this study, the diagnosis of pneumonia by the physicians in charge was twofold increased in comparison to the retrospective diagnosis made by the three experts. This overestimation by the physician may also explain the lack of difference of total antibiotic consumption and antibiotic-free days in the high-risk subgroup, although there is a trend. Nevertheless, the lack of consistent beneficial effect in the whole population raises the question of the futility of this treatment outside perhaps highrisk patients. A second limitation is the potential bias of interpretation which may have resulted from a high rate of very late onset pneumonia ( $>10$ days), long after the peak of postoperative sternal pain known to occur during the first $48 \mathrm{~h}$, and thus long after the potential beneficial effect of continuous infusion of L-bupivacaine. However, we observed that most pneumonia occurred during the first 10 days. Third, $7.3 \%$ of patients did not receive the intervention as randomized, which may also limit the power of the study. However, the per-protocol analysis confirmed the results of the intention-to-treat analysis. Lastly, the catheter may be in an inadequate sternal position and/or the dose of L-bupivacaine administered may be insufficient. Nevertheless, beneficial effect of local anesthetic was found with sternal wound infusion, at lower rate of infusion [14-16]. Nevertheless, those monocentric studies investigated samples size of less than 30 patients in total. This underlines the importance of conducting large double-blind multicenter trials, especially when they focus on analgesia.

\section{Conclusion}

In patients undergoing cardiac surgery with sternotomy and $\mathrm{CPB}$, local anesthetic continuous wound infusion failed to decrease the incidence of postoperative pneumonia. These findings do not support the routine use of continuous wound infusion of L-bupivacaine in this indication. Nevertheless, further studies should investigate its potential beneficial effect in high-risk patients.

\section{Electronic supplementary material}

The online version of this article (https://doi.org/10.1007/s00134-018-5497-x) contains supplementary material, which is available to authorized users.

\begin{abstract}
Author details
${ }^{1}$ Department of Anesthesiology and Critical Care Medicine, Pitié-Salpêtrière Hospital, Institut de Cardiologie, Réanimation de Chirurgie Cardiaque, Sorbonne Université, UMR INSERM 1166, IHU ICAN, Assistance Publique-Hôpitaux de Paris (AP-HP), 47-83 Boulevard de l'Hôpital, 75013 Paris, France. ${ }^{2}$ Department of Anesthesiology and Critical Care Medicine, Hôpital Européen Georges Pompidou, Université Paris-Descartes, Sorbonne Paris Cité, AP-HP, Paris, France. ${ }^{3}$ Biology of Cardiovascular Diseases and Department of Anesthesiology and Critical Care, Magellan Medico-Surgical Center, University of Bordeaux, INSERM, UMR 1034, Bordeaux, France. ${ }^{4}$ Department of Anesthesiology and Critical Care Medicine, Hôpital Bichat-Claude Bernard, Unité INSERM U1 148 (Laboratory for Vascular Translational Science), Université Paris-Diderot, Sorbonne Paris Cité, AP-HP, Paris, France. ${ }^{5}$ Department of Cardiovascular and Thoracic Surgery, Pitié-Salpêtrière Hospital, Sorbonne Université, UMR INSERM 1166, IHU ICAN, Assistance Publique-Hôpitaux de Paris (AP-HP), Paris, France. ${ }^{6}$ Université Claude Bernard Lyon 1, Inserm U1060, Department of Anesthesiology and Critical Care Medicine, Hôpital Louis Pradel, Hospices Civils de Lyon, Lyon, France. ${ }^{7}$ Department of Emergency Medicine and Surgery, Pitié-Salpêtrière Hospital, Sorbonne Université, UMR INSERM 1166, IHU ICAN, Assistance Publique-Hôpitaux de Paris (AP-HP), Paris, France. ${ }^{8}$ Department of Cardiovascular and Thoracic Surgery, Hôpital Européen Georges Pompidou, Université Paris-Descartes, Sorbonne Paris Cité, AP-HP, Paris, France. ${ }^{9}$ Department of Cardiovascular and Thoracic Surgery, Magellan Medico-Surgical Center, University of Bordeaux, INSERM, UMR 1034, Bordeaux, France. ${ }^{10}$ Department of Biostatistic, Public Health and Medical Information, Pitié-Salpêtrière Hospital, Sorbonne Université, Assistance Publique-Hôpitaux de Paris (AP-HP), Paris, France.
\end{abstract}

\section{Acknowledgements}

We thank Dr David Baker, DM, FRCA (Emeritus Consultant Anesthesiologist, Department of Anesthesiology and Critical Care, Hôpital Necker-Enfants Malades, APHP, Paris, France) for editing the article. STERNOCAT Investigators: Hôpital Pitié-Salpêtrière, AP-HP, and Sorbonne Université, Paris, France: Louis Puybasset, MD, PhD, Dimitri Margetis, MD, PhD, Guillaume Lebreton, MD, Mojgane Laalie, MD, PhD; Théodoro Barreda, MD, Cossimo D’Alessandro, MD; Hôpital Européen Georges Pompidou, Paris: Marie-Fazia Boughenou, MD, Alain Bel, MD, Jérôme Jouan, MD, Leonara Du Puy Montbrun, MD, Philippe Menasché, MD, PhD; Magellan Medico-Surgical Center, Bordeaux: Astrid Quessard, MD.

\section{Author contributions}

Drs JA and DH had full access to all of the data in the study and take responsibility for the integrity of the data and the accuracy of the data analysis. Concept and design: $A B, B C, A O, D L, J-L F, D H, B R, J A$. Acquisition of data: all authors. Analysis, or interpretation of data: $A B, B R, A L, D H, J A$. Drafting of the manuscript: AB, BR, JA. Critical revision of the manuscript for important intellectual content: All authors. Statistical analysis: AL, DH. Obtained funding: JA. Administrative, technical, or material support: $\mathrm{DH}$. Supervision: JA, DH.

\section{Funding}

The STERNOCAT study was funded by the French Ministry of Health (Programme Hospitalier de Recherche Clinique National, P100107) and sponsored by Assistance Publique-Hôpitaux de Paris (AP-HP). Baxter provided multiperforated wound catheters, Abbott France provided L-bupivacaine, and Wym France provided elastomeric pumps, all free of charge.

\section{Compliance with ethical standards}

\section{Conflicts of interest}

All authors have completed and submitted the ICMJE Form for Disclosure of Potential Conflicts of Interest. No disclosure was reported. 


\section{Role of the funder/sponsor}

Baxter, Abbott France, and Wym France Orion Pharma had no role in the design and conduct of the study, collection, management, analysis, and interpretation of the data; preparation, review, or approval of the manuscript; and decision to submit the manuscript for publication.

\section{Received: 26 August 2018 Accepted: 4 December 2018} Published online: 7 January 2019

\section{References}

1. Eljezi V, Imhoff E, Bourdeaux D et al (2017) Bilateral sternal infusion of ropivacaine and length of stay in ICU after cardiac surgery with increased respiratory risk: a randomised controlled trial. Eur J Anaesthesiol 34:56-65

2. Hulzebos EH, Helders PJ, Favié NJ, De Bie RA, Brutel de la Riviere A, Van Meeteren NL (2006) Preoperative intensive inspiratory muscle training to prevent postoperative pulmonary complications in high-risk patients undergoing CABG surgery: a randomized clinical trial. JAMA 296:1851-1857

3. Scott NBL, Turfrey DJ, Ray DA, Nzewi O, Sutcliffe NP, Lal AB, Norrie J, Nagels WJ, Ramayya GP (2001) A prospective randomized study of the potential benefits of thoracic epidural anesthesia and analgesia in patients undergoing coronary artery bypass grafting. Anesth Analg 93:528-535

4. Ailawadi G, Chang HL, O'Gara PT et al (2017) Pneumonia after cardiac surgery: experience of the National Institutes of Health/Canadian Institutes of Health Research Cardiothoracic Surgical Trials Network. J Thorac Cardiovasc Surg 153:1384-1391

5. Ng CS, Wan S, Yim AP, Arifi AA (2002) Pulmonary dysfunction after cardiac surgery. Chest 121:1269-1277

6. Gao F, Yang LH, He HR et al (2016) The effect of reintubation on ventilatorassociated pneumonia and mortality among mechanically ventilated patients with intubation: a systematic review and meta-analysis. Heart Lung 45:363-371

7. Sasseron AB, Figueiredo LC, Trova K et al (2009) Does the pain disturb the respiratory function after open heart surgery? Rev Bras Cir CardiovasC 24:490-496

8. Baumgarten MC, Garcia GK, Frantzeski MH et al (2009) Pain and pulmonary function in patients submitted to heart surgery via sternotomy. Rev Bras Cir Cardiovasc 24:497-505

9. Wynne R, Botti M (2004) Postoperative pulmonary dysfunction in adults after cardiac surgery with cardiopulmonary bypass: clinical significance and implications for practice. Am J Crit Care 13:384-393

10. Puntillo K, Max A, Timsit JF et al (2018) Pain distress: the negative emotion associated with procedures in ICU patients. Intensive Care Med 44:1493-1501

11. Devlin JW, Skrobik Y, Gélinas C et al (2018) Clinical practice guidelines for the prevention and management of pain, agitation/sedation, delirium, immobility, and sleep disruption in adult patients in the ICU. Crit Care Med 46:e825-e873

12. Bigeleisen PEL, Goehner N (2015) Novel approaches in pain management in cardiac surgery. Curr Opin Anaesthesiol 28:89-94

13. Bouza E, Hortal J, Munoz P, Pascau J, Perez MJ, Hiesmayr M (2006) Postoperative infections after major heart surgery and prevention of ventilator-associated pneumonia: a one-day European prevalence study (ESGNI-008). J Hosp Infect 64:224-230

14. Dowling R, Thielmeier K, Ghaly A, Barber D, Boice T, Dine A (2003) Improved pain control after cardiac surgery: results of a randomized, double-blind, clinical trial. J Thorac Cardiovasc Surg 126:1271-1278
15. White PF, Rawal S, Latham P et al (2003) Use of a continuous local anesthetic infusion for pain management after median sternotomy. Anesthesiology 99:918-923

16. Chiu KM, Wu CC, Wang MJ et al (2008) Local infusion of bupivacaine combined with intravenous patient-controlled analgesia provides better pain relief than intravenous patient-controlled analgesia alone in patients undergoing minimally invasive cardiac surgery. J Thorac Cardiovasc Surg 135:1348-1352

17. Tirotta CF, Munro HM, Salvaggio J et al (2009) Continuous incisional infusion of local anesthetic in pediatric patients following open heart surgery. Paediatr Anaesth 19:571-576

18. Schulz KF, Altman DG, Moher D, CONSORT Group (2010) CONSORT 2010 Statement: updated guidelines for reporting parallel group randomised trials. BMC Med 8:18

19. Manganas H, Lacasse Y, Bourgeois S, Perron J, Dagenais F, Maltais F (2007) Postoperative outcome after coronary artery bypass grafting in chronic obstructive pulmonary disease. Can Respir J 14:19-24

20. Rock P, Rich PB (2003) Postoperative pulmonary complications. Curr Opin Anaesthesiol 16:123-131

21. Gol MK, Karahan M, Ulus AT et al (1998) Bloodstream, respiratory, and deep surgical wound infections after open heart surgery. J Card Surg 13:252-259

22. Calandra T, Cohen J (2005) The international sepsis forum consensus conference on definitions of infection in the intensive care unit. Crit Care Med 33:1538-1548

23. Leone M, Bouadma L, Bouhemad B et al (2018) Hospital-acquired pneumonia in ICU. Anaesth Crit Care Pain Med 37:83-98

24. Van Wingerden JJ, Ubbink DT, Van Der Horst CM, De Mol BA (2014) Poststernotomy mediastinitis: a classification to initiate and evaluate reconstructive management based on evidence from a structured review. J Cardiothorac Surg 9:179

25. Amour J, Birenbaum A, Langeron O et al (2008) Influence of renal dysfunction on the accuracy of procalcitonin for the diagnosis of postoperative infection after vascular surgery. Crit Care Med 36:1147-1154

26. Casagrande JT, Pike MC (1978) An Improved approximation formula for calculating sample sizes for comparing two binomial distributions. Biometrics 34:483-486

27. Jakobsen JC, Gluud C, Wetterslev J, Winkel P (2017) When and how should multiple imputation be used for handling missing data in randomised clinical trials - a practical guide with flowcharts. BMC Med Res Methodol 17:162

28. Rubin DB (1987) Multiple imputation for nonresponse in surveys. Wiley, New York

29. Bang J, Kim JU, Lee YM et al (2011) Spinal epidural hematoma related to an epidural catheter in a cardiac surgery patient - a case report. Korean J Anesthesiol 6:524-527

30. Rosen DA, Hawkinberry DW, Rosen KR, Gustafson RA, Hogg JP, Broadman LM (2004) An epidural hematoma in an adolescent patient after cardiac surgery. Anesth Analg 98:966-969

31. Liu SS, Richman JM, Thirlby RC, Wu CL (2006) Efficacy of continuous wound catheters delivering local anesthetic for postoperative analgesia: a quantitative and qualitative systematic review of randomized controlled trials. J Am Coll Surg 203:914-932

32. Ruka E, Dagenais F, Mohammadi S, Chauvette V, Poirier P, Voisine P (2016) Bilateral mammary artery grafting increases postoperative mediastinitis without survival benefit in obese patients. Eur J Cardiothorac Surg 50:1188-1195

33. Toumpoulis IKL, Theakos N, Dunning J (2007) Does bilateral internal thoracic artery harvest increase the risk of mediastinitis? Interact Cardiovasc Thorac Surg 6:787-791 